\title{
ANÁLISIS DE LA EFICIENCIA TERMINAL DE LA MAESTRÍA EN EDUCACIÓN: CAMPO EDUCACIÓN AMBIENTAL DE UPN-MEXICALI *
}

\author{
Jorge Ramírez-Acosta \\ Doctor en Ciencias en el uso, manejo y preservación de los recursos naturales \\ Profesor-Investigador en el programa de Maestría en Educación: \\ Campo Educación Ambiental de la Universidad Pedagógica Nacional-Mexicali \\ biologicas.ramirez@gmail.com
}

\begin{abstract}
Resumen
Por primera vez, en los 18 años que tiene de historia, se analiza la eficiencia terminal del programa de Maestría en Educación: Campo Educación Ambiental de la Universidad Pedagógica Nacional Unidad Mexicali. El análisis del comportamiento de ingreso, deserción (porcentaje de deserción) y graduación (titulación) del posgrado en cuestión, abarca de 1994 a 2012, obteniéndose la información para los cálculos de los expedientes del posgrado. Los resultados indican que de las 15 cohortes egresadas están conformadas en mayor proporción por docentes que laboran en Educación Básica. Los porcentajes de eficiencia terminal que registra la Maestría están por debajo de lo que se reporta en el nivel de posgrado en México, lo que al parecer está relacionado con la poca experiencia de los estudiantes y egresados para realizar trabajos de investigación, la falta de asesoría, y el sistema de valoración donde laboran. El incremento de la matrícula de profesionistas con formación universitaria sugiere que utilizan el posgrado en educación para incorporarse y/o establecerse como docentes, no es su objetivo principal formarse en el campo de lo ambiental, fenómeno que puede definirse como credencialización pedagógica.
\end{abstract}

Palabras clave: Eficiencia Terminal, Formación Ambiental, Credencialización, Investigación, Posgrado

\footnotetext{
Abstract

The paper analyzes for the first time in 18 years that has been operating the terminal efficiency of Master of Education: Environmental Education, from Universidad Pedagógica Nacional, Mexicali, México. The analysis of the entrance, desertion (desertion rates) and graduation (obtaining grade) of the graduates students in question includes from 1994 to 2012. The results showed to date has graduated 15 generations formed a greater proportion of teachers working in basic education, indicate that the initial formation of students combined with the system in which they work, the limited experience in research labor and the lack of guidance, have historically influenced
}

the low terminal efficiency that records the master program in question. The increase in registration of university-educated professionals outside the education system suggests who attend the postgraduate education to join and / or established as teachers, so their main objective is not be formed in the field of the environmental education, a phenomenon that can defined as teaching credentialing. Of the increase in enrollment of universitytrained professionals is inferred that graduate studies in education will enable them to enter and / or settle in the education system, this phenomenon can be defined as educational credentialing, ignoring the objective that is formation in the field of environmental education. The is no information about the influence of this postgraduate teacher preparation and the development of a new educational practice in different educational levels of Baja California

Key words: Environmental Education, Credentialing Educational, Environmental Training

\section{Introducción}

Las instituciones de educación superior (IES) son el medio para la aplicación de la política educativa y su papel consiste en incorporar a los aspirantes a los estudios superiores y facilitar la realización del proceso formativo, que es lo que finalmente interesa (Cuéllar-Saavedra y Bolívar-Espinoza, 2006). Por lo tanto, su eficiencia depende principalmente de la proporción de estudiantes que logran egresar y titularse respecto a aquellos que ingresaron, a este indicador se le llama eficiencia terminal (ET) (López-Suárez et al., 2008; Subsecretaría de Educación Pública, 2008). 
La formación docente es un proceso permanente, dinámico, integrado y multidimensional, en el que convergen, entre otros elementos, la disciplina y sus aspectos teóricos, metodológicos, epistemológicos, didácticos, psicológicos, sociales, filosóficos e históricos, para lograr la profesionalización de la docencia (Chehaybar, 2003). Las maestrías y especialidades en el área de educación, principalmente las orientadas a mejorar la calidad de la docencia, han sido la principal herramienta para la profesionalización del docente (Mota, 2006, en Arenas-Castellanos y Fernández de Juan, 2009).

En su carácter de máxima casa de estudios en materia de Educación, a nivel nacional y en Baja California, la Universidad Pedagógica Nacional (UPN) ha sido pionera en el diseño e implementación de programas de formación ambiental a nivel de posgrado con maestrías y especializaciones y de actualización con diplomados, además de vincularse a la comunidad mediante talleres de desarrollo comunitario.

Ejemplo de esto último y con el propósito de aportar soluciones desde el contexto educativo a la problemática ambiental actual, la UPN-Mexicali diseñó y puso en funcionamiento en 1993 el programa de Maestría en Educación: Campo Educación Ambiental (MECEA). En la elaboración de la propuesta académica se involucraron expertos de reconocida trayectoria en el contexto nacional e internacional, quienes en conjunto sostenían una perspectiva social y política de la educación ambiental (Fuentes-Amaya, 2002).

Sus objetivos son contribuir a la búsqueda de soluciones desde el campo educativo a la problemática ambiental y formar educadores/as ambientales capaces de desarrollar una nueva práctica educativa que contribuya al desarrollo de una nueva forma de análisis y enseñanza de lo ambiental. Para esto se hace especial énfasis en el diseño de investigaciones que realicen ejercicios de integración teórica a través de los diferentes ejes que conforman el mapa curricular (UPN, 1993).

\section{El inicio de la formación de educadores ambientales en Mexicali}

Desde su inicio, los primeros trabajos de la Maestría se han desarrollado en un curso propedéutico, cuya base del trabajo es la reflexión, análisis y discusión de contenidos, el objetivo era iniciar un proceso de alfabetización ambiental que contribuya a que los sujetos definan sí éste les proporcionará los elementos teórico-metodológicos necesarios para intervenir el campo de lo ambiental desde el contexto donde ellos habitualmente se desarrollan.

Al inicio la planta académica de la MECEA estuvo apoyada por un grupo multidisciplinario, los cuales provenían tanto de UPN como de instituciones de la región y el país (e.g. Universidad Autónoma de Baja California (UABC), Colegio de la Frontera Norte (COLEF), Centro de Estudios Sobre Medio Ambiente UNAM, y el Programa de las Naciones Unidas para el Medio Ambiente (PNUMA) (Pérez-Martínez, Com. Pers. 2011). En este punto es destacable la diversidad de formas de pensar y propuestas metodológicas que convergieron en un campo nuevo en el tiempo y el espacio donde se ubicaba el proyecto hecho realidad, definido en todo momento por la preocupación genuina de atender la problemática ambiental.

Al respecto González (1998) establece:

"como se puede apreciar la educación ambiental no configura un campo monolítico y cerrado. Por el contrario, se trata de un campo constituido por distintas concepciones sobre lo educativo y ambiental, donde es posible identificar discursos sedimentados que comienzan a cuestionarse y discursos alternativos que buscan re-articular el campo de la educación ambiental."

El egreso de la primera generación de educadores ambientales en el noroeste de México de una Universidad creada para el magisterio le da sentido al objetivo central para lo cual 
fue creada la maestría: formación-actualización de profesores/as en servicio, creación de infraestructura, presencia académica de UPN en el Estado de Baja California y el posicionamiento de esta institución a nivel nacional e internacional, todo en relación al campo ambiental.

Han pasado 18 años desde que inició actividades la MECEA, al momento han egresado 15 cohortes, sin embargo, actualmente no se tiene información que nos permita establecer de qué manera los objetivos de este posgrado se han cumplido, principalmente lo relacionado con la formación de profesores del sistema de educación de Baja California, y esto de qué manera ha contribuido a una nueva práctica docente $y$, por ende, al aporte de soluciones de la problemática ambiental del Estado en general desde el contexto de la educación.

Cuéllar-Saavedra y Bolívar-Espinoza (2006) y Sánchez-Dromundo (2007) consideran a la ET como un factor que refleja la calidad educativa, por lo que se convierte en un indicador importante según la manera cómo se use: como indicador del proceso formativo o del desempeño institucional. Por lo tanto, la graduación como objetivo deja de ser un producto (tesis) para convertirse en un proceso de formación (Sánchez-Dromundo, 2007), que inicia con la incorporación al programa de estudio y culmina con la obtención del grado; por tanto, toca y trasciende todos los procesos y prácticas pedagógicas que vive el estudiante a lo largo de su trayectoria (Arredondo y Santa María, 2004) que lo lleva de la simple capacitación a la formación en el campo de estudio elegido, en sí.

Conforme esto, el objetivo del trabajo es analizar la eficiencia terminal del posgrado a lo largo de su historia, así como los factores que pudieran influir. En este análisis se considera como un indicador de la formación en el campo de lo ambiental resultante del tránsito y egreso de dicho posgrado, por lo cual se plantea la siguiente pregunta: ${ }^{2}$ Existe relación entre la eficiencia terminal de la MECEA y la formación ambiental?

\section{Metodología}

Se trabajó con 436 expedientes que contenían información de la matrícula por generación, año de ingreso, egreso y graduación de la MECEA, desde enero de 1994 hasta enero de 2012. Dicha información fue proporcionada por el área de servicios escolares y la coordinación de la MECEA de la Universidad Pedagógica Nacional, unidad Mexicali. En el caso de aquellos expedientes que carecían de información relacionada a la obtención del grado, o estaba incompleta se obtuvo mediante la consulta de la base de datos de la biblioteca pública localizada en dicha universidad.

Con los datos obtenidos se analizó: 1) Comportamiento de ingreso, egreso (porcentaje de deserción) y graduación (titulación) histórico. El ingreso comprendió aquellos estudiantes que una vez concluido el curso de inducción y fueron aceptados, se inscribieron y asistieron a los cursos que conforman la MECEA. El porcentaje de deserción se calculó considerado el total de egreso en relación al ingreso total y por generación. El análisis de la proporción de graduados (titulados) se hizo de manera general y para cada una de las generaciones egresadas.

2) Formación inicial respecto al ingreso, egreso y graduación. Para el análisis se distinguió cuántos tenían formación normalista y cuántos provenían de una formación profesional diferente a la Escuela Normal. Asimismo, se consideró el total histórico, por generación, y total de egresados en relación al total de graduados.

1 De hecho, la presente reflexión es parte de una respuesta más amplia a la pregunta fundamental que aún queda pendiente de contestar en un nivel mayor de complejidad: ¿La formación ambiental ha modificado la práctica docente y la forma de análisis en torno a lo ambiental en los diferentes niveles del sistema educativo estatal? 
3) Tiempo posterior al egreso para graduarse. Se analizó de manera general y conforme a la formación inicial de cada uno de los egresados el tiempo que les tomó graduarse. Además se calculó la proporción de los egresados que actualmente han agotado el tiempo reglamentario para poder presentar su examen de obtención de grado. En esta misma categoría de análisis se incluyeron aquellos egresados en situación de baja académica (créditos anulados conforme al reglamento).

Para evaluar la ET de la MECEA se trabajó con seis variables (ver Tabla I), en tanto para los cálculos se consideró lo establecido por Cuéllar-Saavedra y Bolívar-Espinoza (2006) y López-Suárez et al. (2008).

\section{Resultados}

\section{Nuevo ingreso, egreso y formación inicial de alumnos/as que ingresan}

A 18 años de su inicio (1994 a 2012), la MECEA registra 17 generaciones, de las cuales 15 han egresado y dos más están en tránsito. La matrícula total en este tiempo suma 436 registros. La generación III (1998-2000) es la que mayor matrícula ha registrado con 59 estudiantes, contrario a las generaciones V y XII que han registrado la menor matrícula (Fig. $1)$.

Con base en la formación inicial de los estudiantes y egresados que conforman y/o han conformado las generaciones atendidas, la mayor proporción (60\%) son egresados de Escuelas Normales y carreras relacionadas con educación (e. g. UPN); el resto (40\%) son profesionistas provenientes de diversas carreras (formación universitaria), predominan los del área de Ingeniería y Arquitectura (14\%) (Fig. 2), se destaca la generación XVII la cual registra, en su mayor proporción, estudiantes con formación universitaria (Fig. 3).

\section{Deserción por generación, y su relación respecto a la formación inicial}

Consideradas las generaciones egresadas en el periodo de tiempo 1996 a 2012, la deserción registrada fue del 29\%. Sin embargo, el análisis de ésta por generación en el mismo periodo de tiempo muestra valores que van de cero en la generación I, hasta un máximo de $75 \%$ en la generación VI (Fig. 4). Es destacable el registro de altos porcentajes de deserción en generaciones conformadas por $>30 \%$ de profesionistas con formación universitaria, de éstas la generación VI fue la que mayor porcentaje de deserción registró (Fig. 5).

\section{Eficiencia Terminal (egreso y graduación)}

Consideradas las 15 generaciones egresadas, el $71 \%$ de egresados cubrió los créditos relacionados con las materias que componen el programa de maestría y recibieron, por lo tanto, el certificado parcial de término; por otra parte, del total de quienes ingresaron en el periodo que se evalúa, sólo el $9.11 \%$ obtuvo su título de posgrado; y conforme al total de egresados sólo el $13 \%$ se ha titulado (Tabla II). Los egresados con formación normalista son los que en menor proporción (34\%) se han graduado (Fig. 6). El análisis de la eficiencia terminal en cada una de las generaciones en relación a la obtención del grado muestra que la generación $\mathrm{XI}$ registró el mayor porcentaje (38\%), en tanto las generaciones VI y XIV son las que menor eficiencia terminal presentan, ya que no registran ningún graduado (Fig. 7).

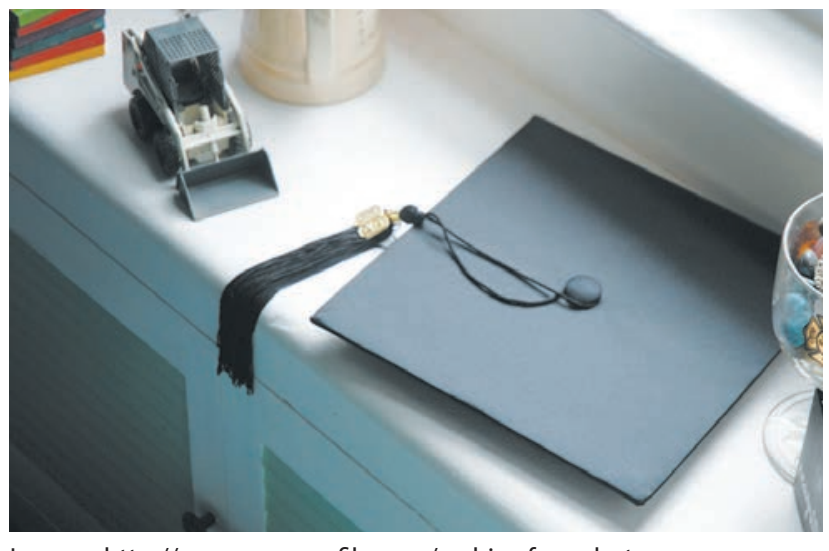

Imagen http://www.morguefile.com/archive free photos 
Tabla I. Definición de variables medidas en el análisis de eficiencia terminal de la Maestría en Educación: Campo Educación Ambiental, unidad Mexicali

\begin{tabular}{|c|c|}
\hline VARIABLE & DESCRIPCIÓN \\
\hline Ingreso (Matrícula) & $\begin{array}{l}\text { Estudiantes que se matricularon y formaron parte de una nueva } \\
\text { generación, medido de } 1994 \text { hasta } 2012\end{array}$ \\
\hline Egresados & $\begin{array}{l}\text { Quienes recibieron certificado de estudios parcial por haber cubierto } \\
\text { los créditos relacionados con las materias que componen el programa } \\
\text { de maestría pero no han presentado su examen para la obtención de } \\
\text { grado }\end{array}$ \\
\hline Formación Inicial & Origen profesional de quienes que se han matriculado en la MECEA \\
\hline Deserción & $\begin{array}{l}\text { Cantidad de estudiantes que abandonaron la maestría de manera } \\
\text { permanente }\end{array}$ \\
\hline Graduados & $\begin{array}{l}\text { Egresados que aprobaron en su examen de obtención de grado y, } \\
\text { por lo tanto, recibieron su acta de examen de grado. En este caso se } \\
\text { mide en lo general, por generación, conforme su formación inicial y el } \\
\text { tiempo que necesitaron para presentar su examen de grado }\end{array}$ \\
\hline Baja académica & $\begin{array}{l}\text { Egresado que, una vez concluidos los créditos en el aula, ha agotado } \\
\text { el tiempo para poder graduarse, conforme lo establece el Reglamento } \\
\text { General de Estudios de Posgrado de la UPN }\end{array}$ \\
\hline
\end{tabular}

Fuente: Autor

Tabla II. Registro total de ingreso, egreso y graduados de la Maestría en Educación: Campo Educación Ambiental, unidad Mexicali en el periodo de 1994 a 2012

\begin{tabular}{|l|c|}
\hline Nuevo ingreso (Total histórico matriculados I-XVII) & 436 \\
\hline Ingreso (Generaciones egresadas I-XV) & 355 \\
\hline Egresados (Generación I-XV) & 271 \\
\hline Graduados (Obtuvieron grado) & 32 \\
\hline Egresados/Ingreso & $71 \%$ \\
\hline Graduados/Ingreso & $9.11 \%$ \\
\hline Graduados/Egreso & $12.9 \%$ \\
\hline
\end{tabular}

Fuente: Autor

2 Para los cálculos sólo consideramos las generaciones que han egresado y no las que aún están en formación. 
Figura 1. Matrícula histórica de la maestría en Educación: Campo Educación Ambiental de UPNMexicali, conforme cada una de las generaciones atendidas de 1994 a 2012

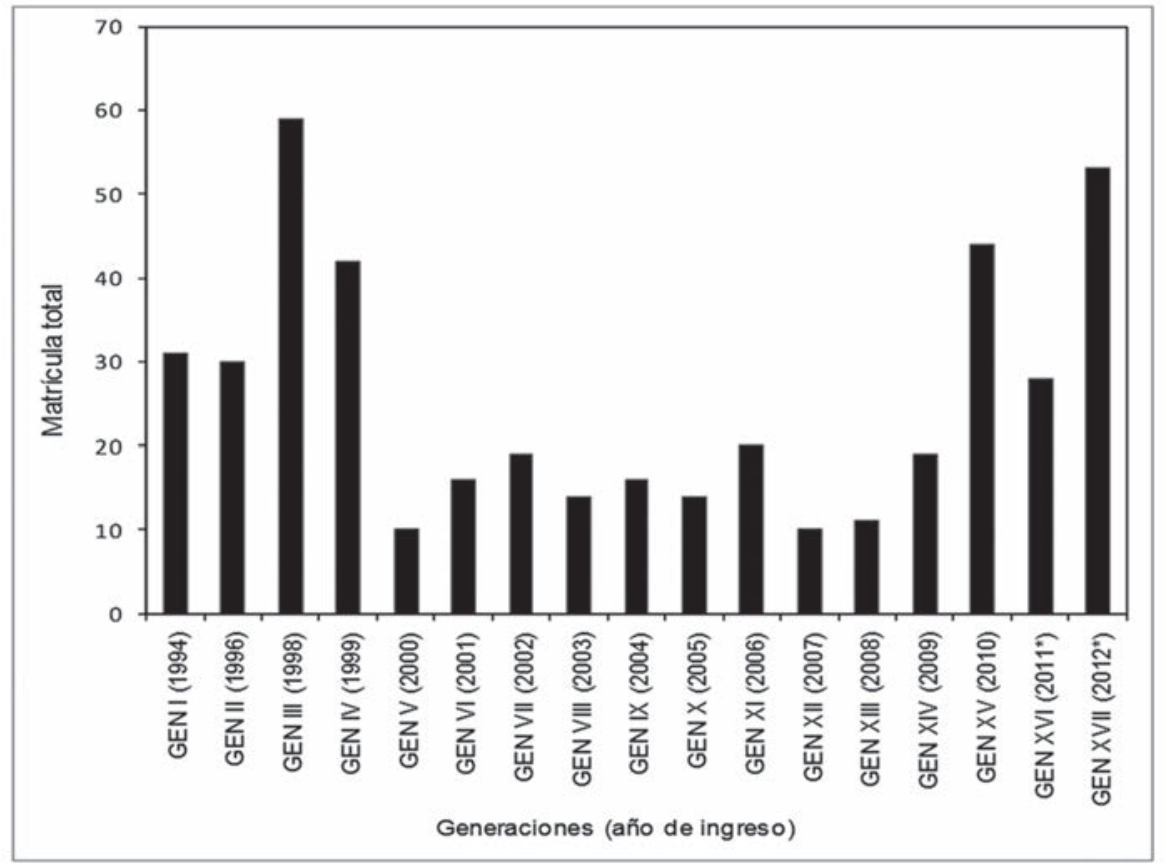

Fuente: Autor

Figura 2. Formación a nivel superior de los estudiantes que conforman o han sido parte de las generaciones atendidas de la maestría en Educación: Campo Educación Ambiental de UPNMexicali, del periodo de 1994 al 2012

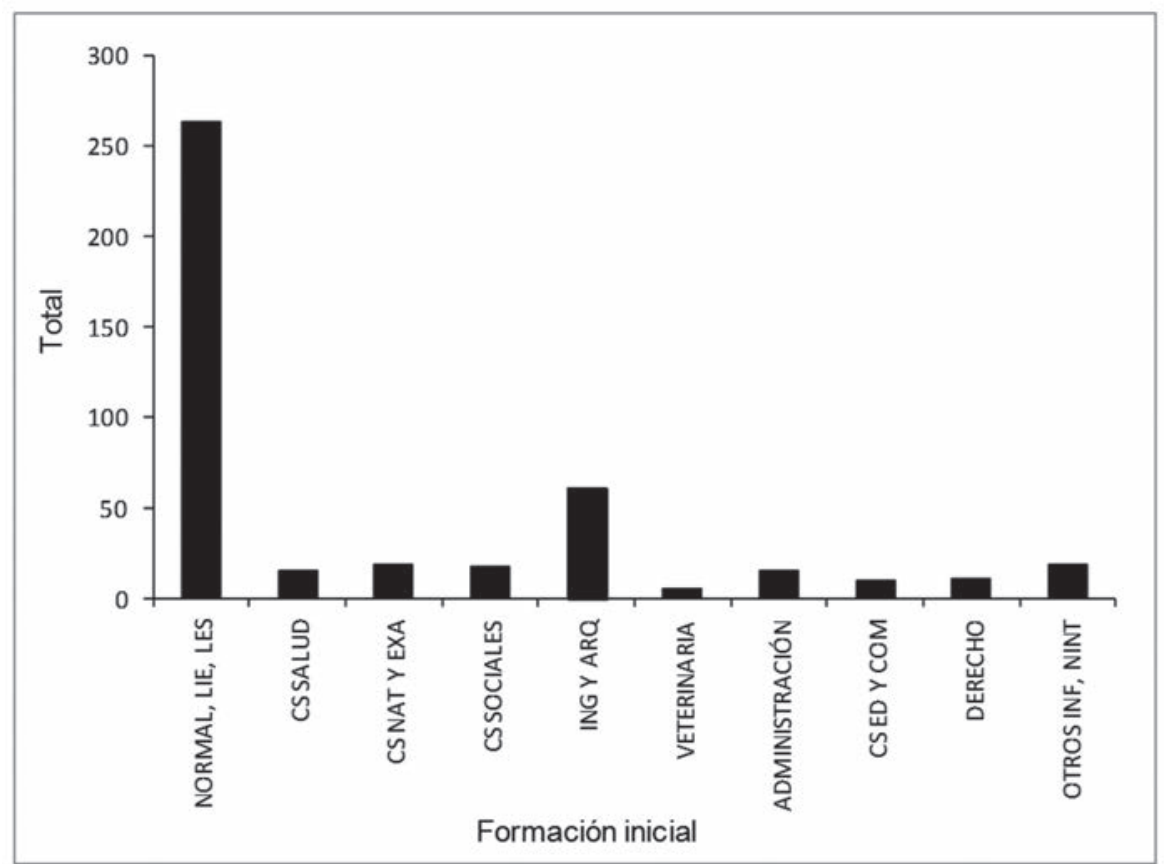


Figura 3. Proporción de estudiantes respecto a su formación inicial en cada una de las generaciones egresadas de la maestría en Educación: Campo Educación Ambiental de UPN-Mexicali

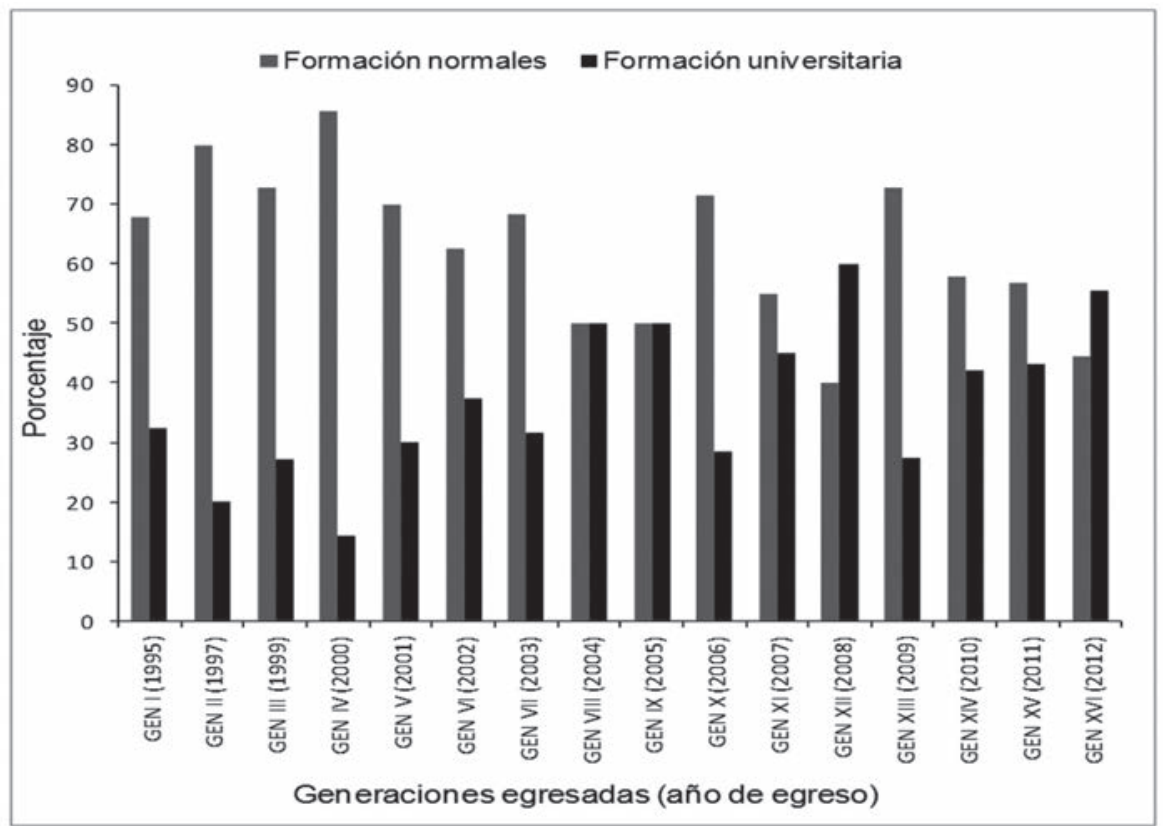

Fuente: Autor

Figura 4. Porcentaje de deserción en cada una de las generaciones egresadas de la maestría en Educación: Campo Educación Ambiental de UPN-Mexicali, del periodo de 1995 al 2012

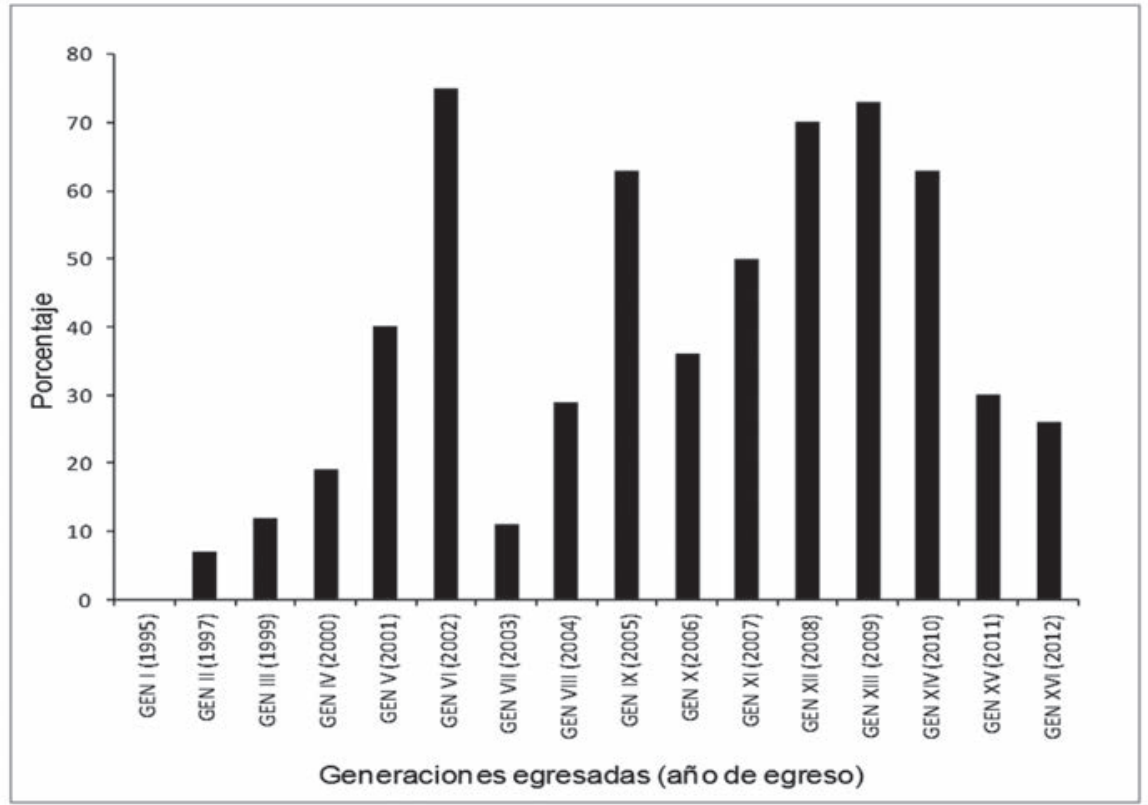


Figura 5. Comportamiento de la deserción considerada la proporción de estudiantes con formación universitaria y normalista en cada una de las generaciones egresadas de la maestría en Educación: Campo Educación Ambiental de UPN-Mexicali, del periodo de 1995 al 2012

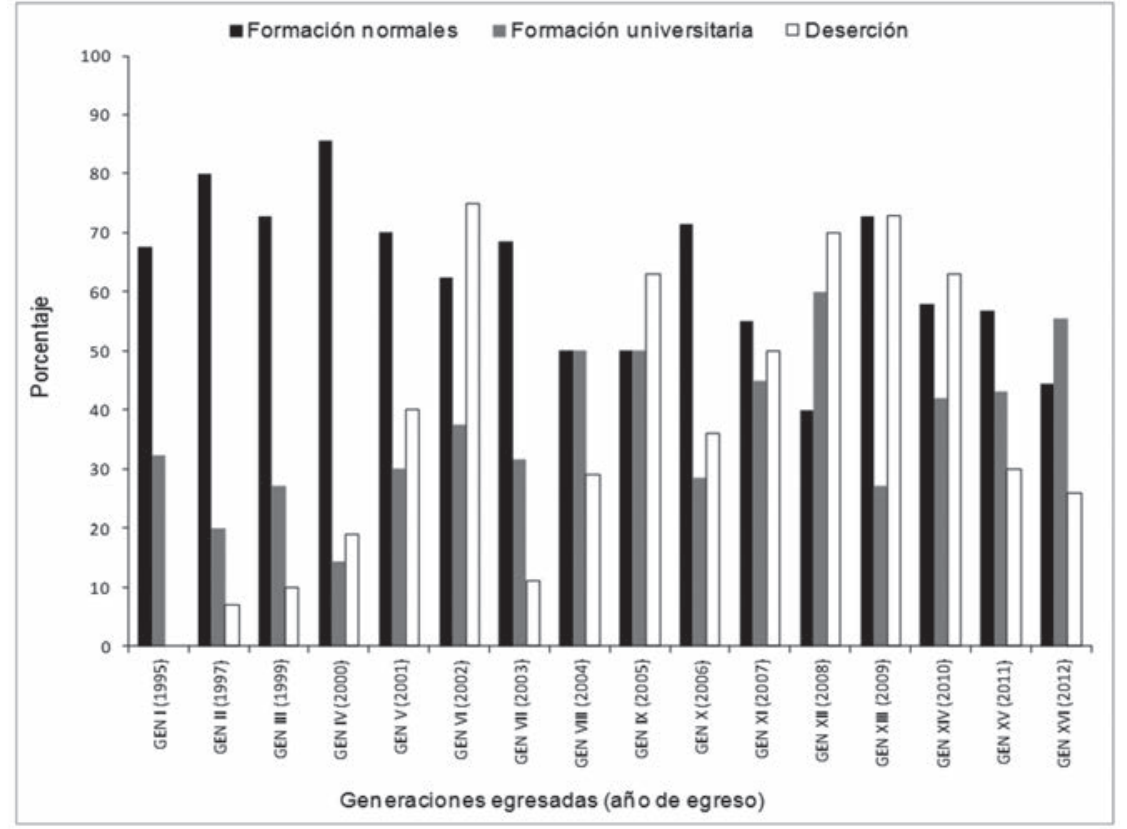

Fuente: Autor

Figura 6. Porcentaje de graduados en la maestría en Educación: Campo Educación Ambiental de UPN-Mexicali, del periodo de 1995 al 2012 considerada su formación inicial

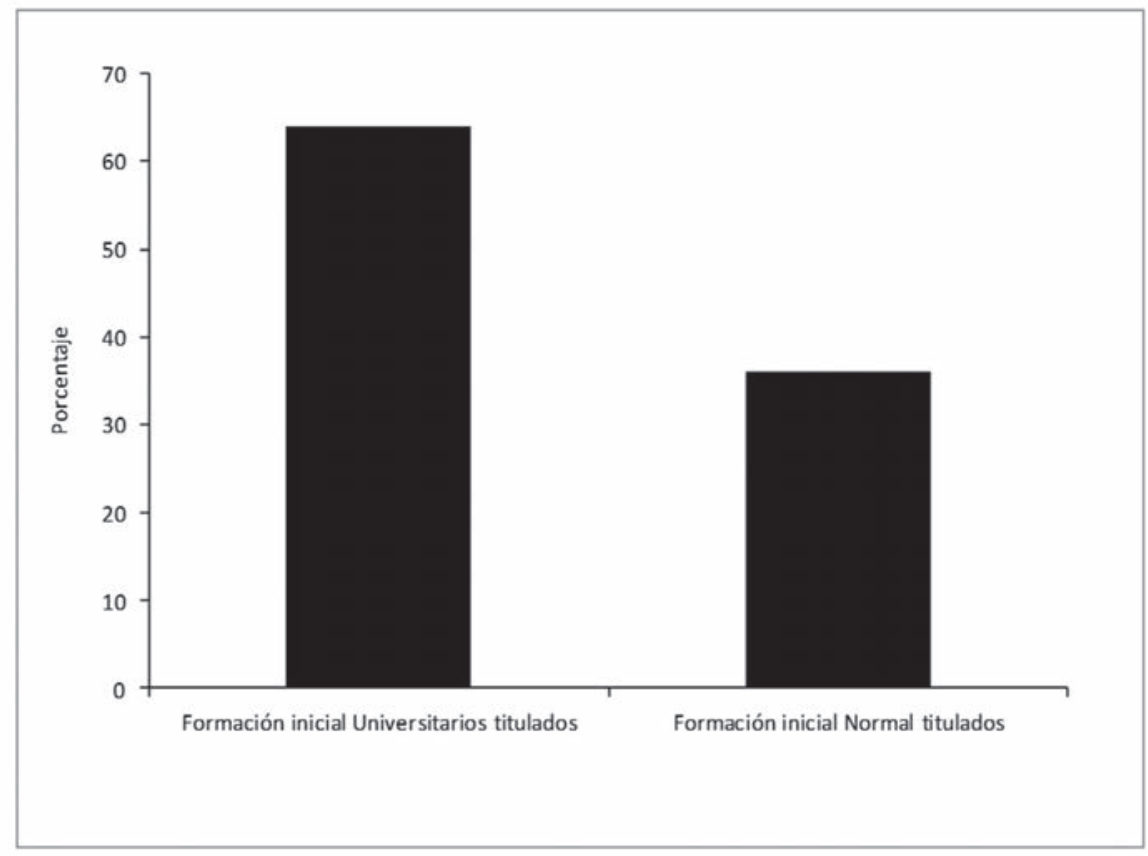


Figura 7. Eficiencia terminal de la maestría en Educación: Campo Educación Ambiental de UPNMexicali, en cada una de las generaciones egresadas del periodo de 1995 al 2012

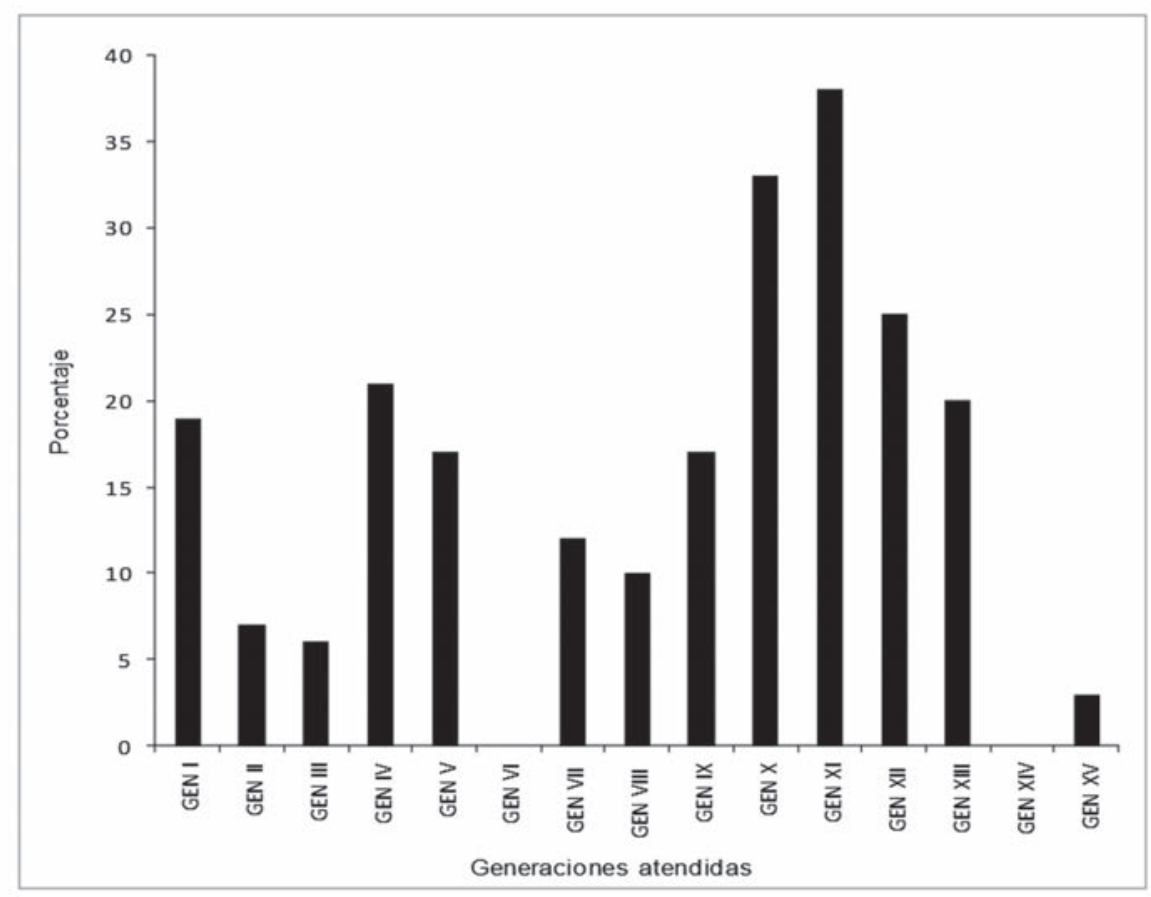

Fuente: Autor

\section{Tiempo reglamentario para graduarse}

El tiempo para graduarse en la MECEA ha sido en promedio de 3.5 años, sin embargo, el mayor porcentaje de egresados graduados (28\%) lo hicieron cuatro años posterior a su egreso. Los egresados graduados con formación normalista, han obtenido el grado en promedio a los 1.2 años, tiempo menor a los graduados con formación universitaria, quienes lo hicieron en promedio a los 2.3 años de haber egresado. Hasta la generación XV se tienen 251 egresados, de este total solo 33 se han graduado, de éstos el $25 \%$ obtuvo su grado después de haber agotado el plazo reglamentario para ello. Quedan 219 aún sin graduarse y conforme al Reglamento de Posgrado, 177 (egresados de las generaciones I a la XI), ya han excedido el tiempo límite, de estos, previo a la obtención del grado 165 deben revalidar el total de créditos de la maestría; seis de la generación $X$ deben revalidar el $75 \%$ de los créditos, y seis más de la generación XI deberán revalidar el 25\%; los 42 restantes (corresponden a las generacio- nes XII a la XV), aún están dentro del tiempo reglamentario para graduarse (Tabla III; Fig. 8) ${ }^{3}$.

\section{Discusión}

\section{Matrícula}

La matrícula histórica de la MECEA permite identificar tres etapas, la primera de éstas de 1994 a 1999 caracterizada por altos registros, que coincide con el tiempo donde lo ambiental como tema emergente y como campo formativo era una oferta atractiva, considerado que a nivel mundial las condiciones anímicas en relación a la temática eran alimentadas por la inercia de la recién terminada Reunión Cumbre de Río, en 1992.

3 Cabe mencionar que si bien el Reglamento vigente establece con claridad que el límite de tiempo que tiene el egresado para obtener el grado será acorde al instructivo del posgrado, sin dejar de considerar lo que hace varias décadas se ha establecido en muchas universidades del mundo, y por la vía normativa de los usos y costumbres, que sea el máximo órgano normativo del posgrado de cada institución el que determine la pertinencia o no de aceptar la graduación extemporánea. Con esto se ha salvaguardado la vigencia de derechos del egresado, permitiéndole completar el ciclo de estudios de este nivel, sin que tal medida constituya perjuicio alguno para alguien (Molina-Hernández, 2012, comunicación personal). 
Tabla III. Tiempo reglamentario por generación para graduarse de Egresados de la maestría en Educación: Campo Educación Ambiental en UPN-Mexicali

\begin{tabular}{|l|c|c|c|c|}
\hline Generación que cursó & Año de Egreso & $\begin{array}{c}\text { Término de tiempo legal } \\
\text { para titularse }\end{array}$ & Tiempo anulación 100\% créditos & Egresados no graduados \\
\hline GEN I & 1995 & 1999 & 2001 & 25 \\
\hline GEN II & 1997 & 2001 & 2003 & 26 \\
\hline GEN III & 1999 & 2003 & 2005 & 49 \\
\hline GEN IV & 2000 & 2004 & 2006 & 27 \\
\hline GEN V & 2001 & 2005 & 2007 & 5 \\
\hline GEN VI & 2002 & 2006 & 2008 & 4 \\
\hline GEN VII & 2003 & 2007 & 2009 & 15 \\
\hline GEN VIII & 2004 & 2008 & 2010 & 9 \\
\hline GEN IX & 2005 & 2009 & 2011 & 6 \\
\hline GEN X & 2006 & 2010 & 2012 & 6 \\
\hline GEN XI & 2007 & 2011 & 2013 & 2 \\
\hline GEN XII & 2008 & 2012 & 2014 & 3 \\
\hline GEN XIII & 2009 & 2013 & 2015 & 7 \\
\hline GEN XIV & 2010 & 2014 & 2016 & 30 \\
\hline GEN XV & 2011 & 2015 & 2017 & \\
\hline GEN XVI & 2012 & 2016 & 2018 & \\
\hline GEN XVII & 2013 & 2017 & 2019 & \\
\hline
\end{tabular}

Fuente: Autor

Figura 8. Proporción de egresados no graduados de la maestría en Educación: Campo Educación Ambiental de UPN-Mexicali con créditos anulados y no anulados, del periodo de 1996 al 2011

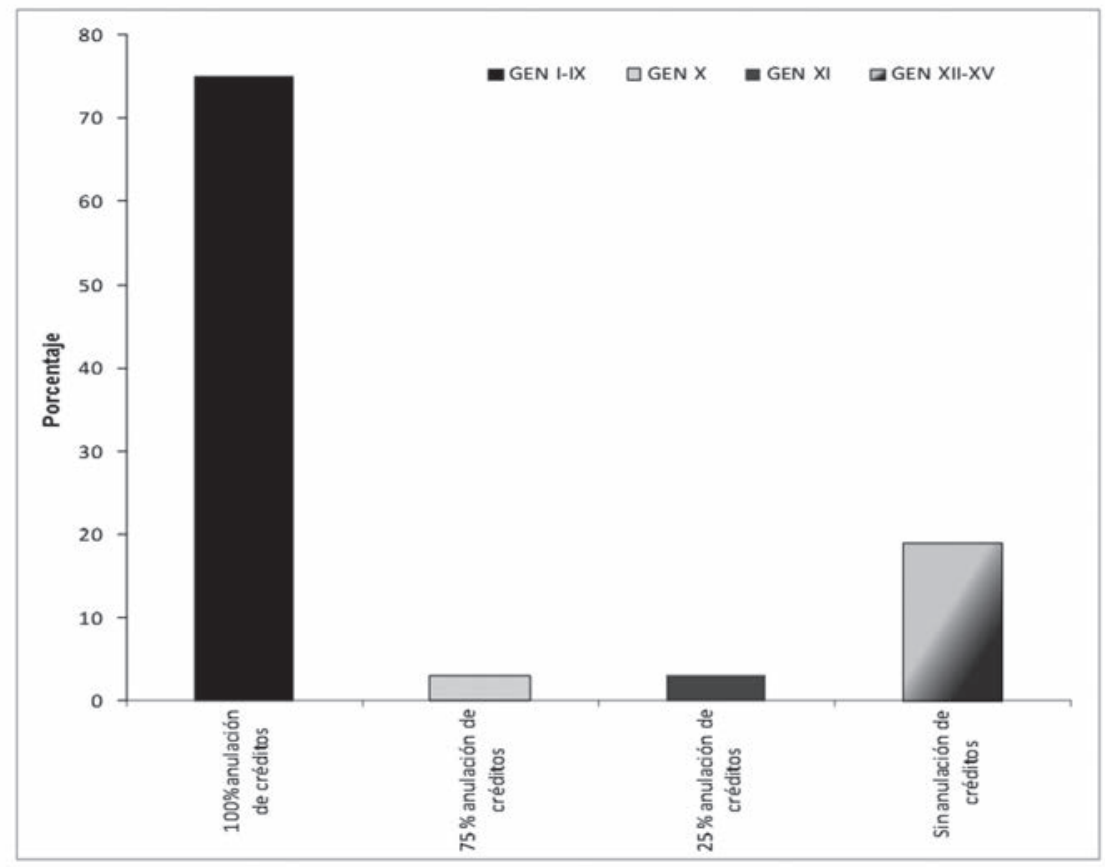


En esta primera etapa, el egreso de la primera generación de educadores ambientales en el noroeste del país en 1996 (cuatro años posterior a dicha Reunión), ilustra, por un lado, la influencia de dicha inercia, y por otro, le da lógica a los objetivos del programa de maestría: la formación-actualización del magisterio en el campo de lo ambiental, además del posicionamiento académico de UPN en dicho campo de intervención en Baja California, a nivel nacional y en el plano internacional.

La alta capacidad de gestión y la dinámica del trabajo académico previa al egreso de la primera generación de la MECEA le permitieron en poco tiempo obtener reconocimientos en materia de formación educativa ambiental en el país y principalmente en el estado de Baja California, además de apoyos del Servicio de Pesca y Vida Silvestre de los Estados Unidos, al seno del Programa de Cooperación para la Conservación de la Biodiversidad México-Estados Unidos. Estos apoyos permitieron trabajar y desarrollar talleres en relación al campo de lo ambiental, hacer estancias en campo y crear infraestructura básica para el funcionamiento del programa de maestría. Se logró además, abrir espacios de discusión como la Red Regional de Educadores Ambientales del Noroeste, e implementar propuestas relacionadas con la capacitación y actualización (e.g. diplomados) basados en lo filosófico (enfoque sistémico desarrollado), lo social (articulación entre lo social y lo natural), y lo metodológico (la interdisciplinariedad en particular), lo esencial de esta propuesta era articular una visión sistémica compleja que incorporaba un diálogo entre las ciencias naturales y las sociales (Fuentes-Amaya 2002). De esto se puede suponer que el posgrado se convirtió en un espacio académico atractivo, lo cual podría explicar los incrementos de la matrícula en las generaciones III y IV.

La segunda etapa está definida por la reducción en la matrícula entre 2000 y 2010, y el incremento de los porcentajes de deserción entre las generaciones V y VI. Esto en su conjun- to podría ser, por un lado, el indicador que lo ambiental como campo de intervención y formación no era una demanda real del magisterio en el tiempo cuando se definió la propuesta académica de la MECEA, considerado que no se hizo un estudio previo de las necesidades de formación del magisterio en el campo en cuestión; y por el otro, el reflejo de la desorganización de la MECEA a la salida del equipo fundador, previo al egreso de la IV generación (Ramírez-Acosta, Obs. Pers., 2005).

La tercera etapa está definida por el incremento en la matrícula a partir del 2010 (generaciones $\mathrm{XV}-\mathrm{XVII})$, esto aparentemente sugiere que el interés por la temática ambiental al interior del contexto educativo se ha "reactivado"; sin embargo, esto debe ser tomado con cautela ya que el solo hecho que se reciba un alto número de solicitudes para el registro y posible ingreso al posgrado no significa que se tiene el interés real, o que el posgrado y su orientación resulte atractivo desde el punto de vista académico. Está claro que, más allá de que se cubran los requisitos que se requieren para el ingreso, un porcentaje importante de los que se matriculan buscan sólo "credencializarse", y no propiamente formarse en el campo de lo ambiental, (Ramírez-Acosta y Molina-Hernández, 2011).

Ejemplo de lo anterior puede ser lo que Molina-Hernández (Com. Pers., 2010) comentan: la generación XV (una de las más numerosas y con alta proporción de profesionistas con formación universitaria) buscaba estudiar un posgrado, pero el campo de lo ambiental no era su prioridad. Esto mismo puede suceder en las generaciones que recién han ingresado, las cuales están conformadas por una alta proporción de estudiantes con formación universitaria, e incluso habría que revisar cuántos de éstos se desempeñan en puestos o áreas donde es necesaria la actualización y formación en el campo de lo ambiental. Por ello, resulta necesario revisar tanto el proceso y el procedimiento de selección como los objetivos del posgrado, 
con el fin de aceptar profesionistas interesados específicamente en la orientación y el tipo de posgrado que se ofrece, tal como lo han hecho en otras instituciones donde se imparten posgrados de temáticas similares (Arias, Com. Pers., 2012; Bedoy-Velázquez et al., 2008).

\section{Formación Inicial y formación ambiental}

Al revisar la formación inicial de las generaciones registradas del 2009 al 2012-1, el incremento de profesionistas con formación universitaria ha sido sostenido, de esto se puede inferir que los estudios de posgrado en educación les facilitarán incorporarse o establecerse en un futuro mediato en el sistema de educación, definido este fenómeno como credencialización pedagógica (Obs. Pers.). No obstante, se debe destacar que como espacio académico la MECEA atiende lo sugerido en la conferencia de Tbilisi (1977) respecto a la necesidad de que los programas de educación ambiental deberían servir también a los profesionales (ingenieros, planificadores urbanos, arquitectos, médicos, docentes, y administradores industriales, entre otros), investigadores y especialistas en materia ambiental, cuyas actividades tengan un impacto directo sobre el medio ambiente (UNESCO, 1978).

Aunado a lo anterior, es importante mencionar que la incorporación de profesionistas que provienen de y se desempeñan en distintos campos del conocimiento ha enriquecido el discurso al interior del aula y en ocasiones del posgrado en general, que potencian la posibilidad que los análisis de la temática ambiental se hagan desde un enfoque integral. Sin embargo, dado que la MECEA está dirigida a profesionistas en educación básica (profesionistas insertos en el sistema magisterial), es necesario revisar las necesidades formativas de aquellos profesionistas insertos en el sector industrial, gubernamental, político, económico y el tercer sector, ya que conocer dichas necesidades permitirá replantear los diferentes ejes que componen la MECEA, pues buscan fortalecer el enfoque integral y la pertinencia como opción formativa que es el posgrado (Ramírez-Acosta y Molina-Hernández, 2011).

\section{Deserción}

El porcentaje de deserción registrada en la MECEA de 1994 al 2010, se encuentra por debajo de lo reportado en el país a nivel de posgrado (32\%) y de posgrados de temáticas similares (31\%) (Bedoy-Velázquez et al., 2008), sin embargo, éste es ligeramente más alto que el $28 \%$ registrado para el estado de Baja California, (ANUIES, 2009), de esto se puede inferir que es parte de la dinámica del nivel en nuestro país, lo cual pudiera ser un indicador de la falta de claridad que se tiene a la hora de decidir por el tipo de propuesta académica aunado a la poca posibilidad de ejercer una vez terminado este proceso de formación.

Sin embargo, el análisis de la deserción en la MECEA es mucho más significativo al revisarse por cada generación, durante las primeras cuatro generaciones (de 1996 a 1999) la deserción varió de cero a 19\% (el promedio fue de $9.4 \%$ ), al parecer lo que influyó en este bajo porcentaje fue el liderazgo de la coordinación de dicho posgrado tanto en lo académico como en la gestión de recursos. Cabe mencionar al respecto, dicho liderazgo y la necesidad de definir una identidad utilitaria en este nuevo campo de intervención pedagógica, motivó a los estudiantes de la primera generación (la cual no registró deserciones) a conformar equipos de trabajo para apoyar los talleres denominados Talleres de la Comunidad en la Comunidad, los cuales se basaban en el diseño e implementación de talleres de educación ambiental (EA) en apoyo a la comunidad en general, además de incorporarse a las tareas docentes de la maestría.

Esto llevó a ser considerado como un programa de posgrado exitoso desde la perspectiva de su capacidad interpelatoria (FuentesAmaya, 2002), con recursos considerables, con un campo de intervención definido y, por ende, 
con altas posibilidades de ejercer al egresar, aunado al contacto con especialistas en el campo tanto a nivel nacional como internacional.

Los incrementos en la deserción a partir de la generación $\mathrm{V}$ y $\mathrm{VI}$, pudieran estar relacionados con dos aspectos fundamentales: 1) la desorganización de la MECEA a la salida del equipo fundador, con lo cual se perdió la experiencia de gestión para la obtención de recursos, la creación de infraestructura básica (e.g. biblioteca especializada), y contacto con especialistas que enriquecían el espacio, todo esto en respaldo a las primeras cuatro generaciones; y 2) la poca o nula experiencia en el campo de la educación ambiental de la planta docente que se conformó para atender las generaciones entrantes la V y VI.

Por otra parte, cabe la posibilidad de que los porcentajes de deserción pudieran estar relacionados con la formación inicial de los estudiantes, si se considera que de aquellas generaciones en las que hubo una alta proporción de estudiantes con formación inicial universitaria, en seis de ellas se registraron altos porcentajes de deserción (e.g. generación XII), infiriéndose que abandonan el posgrado porque no encuentran respuesta a sus necesidades de formación en el campo de lo ambiental. Desafortunadamente los datos utilizados en este análisis no permiten establecer de manera clara si existe dicha relación, insuficiencia que resalta la importancia de definir los instrumentos de medición pertinentes para revisar a detalle si existe relación entre los niveles de deserción y la formación inicial de los estudiantes.

Otro de los factores que pudieran influir sobre los porcentajes históricos de deserción es el tiempo disponible para atender las tareas de la maestría al considerar que la mayor parte de los estudiantes matriculados son profesores en servicio (algunos laboran doble turno), por lo tanto, atienden los estudios de maestría una vez que han terminado su jornada laboral (Ramírez-Acosta y Molina-Hernández, 2011), por lo cual resulta importante implementar estrategias (e.g. solicitud de becas) que les permitan adecuar horarios y cargas de trabajo para evitar demeritar la cantidad del tiempo que dedican al posgrado.

Aunado a lo anterior, será importante revisar aspectos como la estructura curricular de la MECEA (Ramírez-Acosta y Molina Hernández, 2011), y en su caso adecuarla a las necesidades de formación que imponen las nuevas generaciones; además de implementar acciones que lleven a retomar el liderazgo institucional en el campo de lo ambiental que tenía en sus inicios el programa de maestría (ver logros al inicio de este apartado), además de apoyar los procesos administrativos que permitan captar recursos para crear infraestructura, estancias en campo, y el incremento de personal de tiempo completo, por mencionar algunos.

\section{La eficiencia terminal del programa (gradua- dos)}

Normalmente, la graduación de estudiantes se relaciona con la producción de la tesis y la obtención del grado. Esta postura olvida que, para que potencialmente sea posible este trabajo, es necesario considerar todos los factores, las prácticas y los procesos que le permiten al estudiante lograr la certificación (Sánchez-Dromundo, 2007).

Los datos más recientes muestran que el índice de ET (egresados graduados) a nivel de posgrado en México se ubica alrededor del 64 $\%$, mientras que para el estado de Baja California a nivel de maestría la ET alcanza el 91\% (ANUIES, 2011), asimismo, la ET de posgrados con temática similar llegan hasta el $44.5 \%$ (Bedoy-Velázquez et al., 2008) y $19.33 \%$ (Arias, com. pers. 2012).

Considerado que la MECEA es un posgrado que basa su propuesta en la investigación (UPN, 1993), los pobres porcentajes de ET, tanto a nivel general $(9.1 \%)$ como en cada una de las generaciones (15\% en promedio), sugieren 
que los objetivos planteados en el diseño del programa no se han cumplido. Al hacer el comparativo de los diferentes porcentajes permite definir a la MECEA como un posgrado de bajo perfil que dista mucho de los estándares mínimos de eficiencia, índices que impactan en la calidad del posgrado, con el consiguiente desperdicio de recursos físicos, económicos y humanos.

Por otra parte, de los diferentes análisis se puede inferir que al menos tres factores han influido históricamente en la pobre ET de la MECEA: 1) la formación inicial de los estudiantes, aunada al sistema donde laboran, 2) la ausencia histórica en la aplicación estricta del reglamento de posgrado, 3) la falta de asesoría en su trayectoria académica en el posgrado para completar su proceso de formación en el campo de lo ambiental.

Relacionando la formación inicial y el alto porcentaje de egresados no graduados, se infiere que aquellos (tanto normalistas como universitarios) que están insertados en el sistema magisterial de enseñanza (i.e. educación básica, media superior), no se gradúan inmediatamente al término del posgrado -y no lo harán- porque mientras fueron estudiantes combinaron su tiempo de trabajo con el que le dedicaban a la atención de los cursos de maestría.

Esto evita en parte que dediquen tiempo a las actividades de investigación y dado que la única opción para titularse consiste en la elaboración de una tesis, lo cual implica un trabajo terminal escrito, la complejidad de su elaboración y el tiempo que el egresado debe dedicarle constituyen los más fuertes obstáculos para la titulación (Legorreta, 2001), pues su presentación se difiere, de tal manera que al contraer otros compromisos, se titula tardíamente y, en algunos casos, nunca lo hace.

Aunado a lo anterior está la escasez de asesores expertos o bien a la deficiente asignación de los temas que se desarrollarán o se han pre- sentado para la obtención del grado, considerado el perfil de los académicos que conforman la MECEA. Como ejemplo cabe mencionar que de los 30 temas registrados y presentados, el $43 \%$ (13 temas diferentes) han sido dirigidos por un mismo académico; en contraste, la planta académica históricamente ha estado conformada por 14 docentes, y de estos existen casos que nunca han dirigido la elaboración de un trabajo recepcional (Obs. Pers., 2012).

Por otro lado, está el sistema de valoración magisterial, donde por sólo presentar ante la instancia de acreditación o valoración del desempeño docente el certificado de estudios de posgrado, se les asigna 75 puntos de 100 posibles en la puntuación escalafonaria, esto les permite mantenerse o lograr un buen lugar dentro del catálogo de escalafón y con el tiempo ascender a otro puesto (Reglamento Comisión Mixta de Escalafón, 1990; Auyón y Durazo, 2003).

Por lo tanto, elaborar un documento posterior al egreso para graduarse (i.e. obtener el grado de maestría) es poco probable, dado que la diferencia en puntos escalafonarios no es significativa (i.e. 25 puntos por el grado, BandaLuna, Com. Pers., 2012), y en caso de ser baja académica por no graduarse en tiempo y forma la puntuación obtenida no se anula, esto no lo ven como riesgo de retroceder en lo laboral, por lo cual deja de ser prioridad culminar el proceso de formación a nivel posgrado.

En el caso de los egresados ajenos al sistema de acreditación o valoración magisterial que laboran o se desempeñan en el sector industrial, gubernamental, o que trabajan por su cuenta, se puede suponer que obtener el grado no les reditúa algún estímulo económico, o ascensos laborales, por lo cual no existe algo que los obligue a graduarse (i.e. sistema de valoración académica).

Por otra parte, la mayor proporción de graduados con formación universitaria podría ex- 
plicar la mayor experiencia en relación a cómo hacer investigación, además que la obtención del grado académico les asegura o posibilita participar en las promociones de ascenso, ocupar puestos claves (e.g. direcciones), obtener mejores calificaciones acorde a la valoración de su desempeño docente, o bien integrar cuadros académicos ya sea para desarrollar tareas de investigación o impartir cursos relacionados con la temática del posgrado en el sistema en el cual laboran.

Otro factor identificado, es la omisión histórica en relación a los tiempos estipulados para graduarse tal como se establece en el Reglamento General de Estudios de Posgrado de UPN (1999) en su Artículo 86, donde se establece que el egresado podrá presentar la tesis para la obtención del grado en un tiempo que no exceda el doble de lo que señale cada una de las ofertas de posgrado, si el plan de estudios de cada oferta así lo admite.

Asimismo, en el artículo 87 de dicho reglamento se menciona que posterior a ese plazo por cada semestre transcurrido se eliminará acumulativamente el $25 \%$ de los créditos obtenidos hasta anular la totalidad; en cada caso se tendrán que volver a cursar las materias correspondientes antes de presentar la tesis respectiva. Conforme las indagaciones hechas, se supone que el tiempo máximo para graduarse es de cuatro años posteriores al egreso, pero no conocían sí posterior a ese tiempo procedía la anulación de créditos.

¿Cuáles serían las implicaciones de la aplicación literal de la norma vigente? A los egresados de las primeras nueve generaciones (no graduados hasta el momento), se les tendría que notificar la anulación del 100\% de sus créditos y hacer de su conocimiento que en caso de solicitar examen de grado tendrían que revalidar la totalidad de los créditos académicos conforme a Reglamento, o bien proceder a su baja académica definitiva. Los egresados no graduados de las generaciones X y XI tendrían al momento el 75 y $25 \%$ de los créditos anulados respectivamente, y en todos los casos tendrían que revalidar los créditos (i.e. matricular los cursos correspondientes) para graduarse (Reglamento General de Estudios de Posgrado, 2012).

Con respecto a los graduados (25\%) que obtuvieron el grado posterior al tiempo estipulado en el reglamento, se les debió hacer de su conocimiento la situación académica previo a su examen, por lo que se infiere que no se les notificó la irregularidad académica en que incurrieron. Posiblemente fue por desconocimiento o con el fin de promover la graduación, sin embargo, de continuarse presentando el desorden académico que los datos indican, será poco probable que la MECEA eleve su eficiencia terminal $y$, por ende, su calidad académica en el campo de lo ambiental.

El factor determinante para el éxito de este trayecto es una adecuada integración académica y social del estudiante. Ésta se valora por la frecuencia e intensidad de los intercambios entre los sujetos, elementos que contribuyen a la circulación de conocimientos y a la integración de este tejido de relaciones y vínculos. Este entramado se considera como la vida académica, y se afirma que cuanto más frecuentes e intensos sean los intercambios entre los agentes participantes, mayor formación y eficiencia terminal habrá (Arredondo y Santa María, 2004).

Molina et al. (2001) mencionan que la formación en investigación a nivel posgrado es una parte fundamental del currículo, ya que en teoría, al menos, el propósito fundamental es que los estudiantes se conviertan en investigadores de su propia práctica. Sin embargo, la poca experiencia de los profesionistas con formación normalista para hacer investigación, la posible falta de claridad respecto a lo que buscan obtener de este posgrado, y lo que quieren indagar en este campo (Ramírez-Acosta y Molina-Hernández, 2011).y la ausencia de mecanismos que orienten y asesoren a los estudiantes 
indistintamente de su formación inicial desde el propedéutico y durante su trayectoria en el posgrado, incluso posterior al egreso, para el diseño e implementación de propuestas de intervención sobre una problemática en el campo de la educación ambiental, ocasiona la dispersión de éstos.

Jaik-Dipp y Quiroga-Sánchez (2009) encontraron que estudiantes de posgrado en gestión ambiental establecen que al no tener conocimiento o experiencias previas en relación a un tema en específico, no le encuentran significado a los nuevos conocimientos y, por lo tanto, se les dificulta su organización. En el mismo sentido, Ausubel (1983; citado por Jaik-Dipp y Quiroga-Sánchez, 2009) manifiesta que cuando los conocimientos previos interactúan con la nueva información, se modifican unos y otros y dan paso a aprendizajes significativos, y que una condición para que se dé la significancia es que el estudiante tenga los conocimientos previos que le permitan acceder a los nuevos.

Por lo cual se debe exigir desde el propedéutico a cada aspirante el proyecto que va a desarrollar $y$, desde ese momento asignar tutores para que oriente al potencialmente estudiante sobre cómo abordar y desarrollar la propuesta a lo largo de los cuatro semestres. Aunado a la reactivación de las diversas estrategias que para solucionar el rezago e incrementar la eficiencia terminal se han implementado a lo largo del tiempo, algunas de éstas serían los encuentros semestrales de investigación en educación ambiental, el objetivo es dar seguimiento a los avances registrados del documento recepcional durante los cuatro semestres y previo al egreso; el diplomado en investigación aplicado a la educación ambiental y el taller de asesoría para la elaboración de tesis, ambos dirigidos principalmente a egresados que buscan completar su ciclo de formación en el nivel de posgrado.

Lo anterior asociado a un sistema de evaluación y el seguimiento del aprovechamiento de cada uno de los estudiantes en cada uno de los semestres permitirá, por un lado, conocer de manera precisa el crecimiento académico de los estudiantes y, por otro, establecer el avance en la propuesta de intervención previo al egreso lo que, se supone, reducirá la idea que muchos tienen, el tan sólo credencializarse en el campo de la educación ambiental y de la educación en general.

\section{Conclusiones y Sugerencias}

Hasta antes de este trabajo no se tenía información sistematizada de la eficiencia terminal de la MECEA, se espera que sirva como referente para evaluar el posgrado en general de UPN-Mexicali, con el fin de incidir en la calidad académica y hacer más eficientes los procesos internos de la institución. Dado los altos porcentajes de deserción, se requiere elevar el nivel de atención a los estudiantes durante su trayecto en el posgrado, por lo cual se debe evitar masificar la matrícula de nuevo ingreso y, a su vez, la convocatoria a un nuevo proceso de admisión tiene que ser al término de cada generación, esto mejorará los procesos de planeación administrativa y de atención académica, de otra manera sólo se promueven procesos de credencialización sin aportes significativos al campo de la educación ambiental. En el mismo sentido, se requiere establecer un programa de seguimiento a egresados para conocer por un lado cómo la MECEA ha influido en la formación ambiental y el quehacer pedagógico del profesor de educación básica y, por otro, evaluar el aporte académico hacia los profesionistas ajenos al sistema educativo (e.g. sector industrial, gubernamental y del tercer sector), el conocimiento de esto permitirá fortalecer el enfoque integral y la pertinencia como opción formativa que es el posgrado. Considerada la poca experiencia de los estudiantes con formación normalista y similar a ella para el desarrollo de tareas de investigación, se debe solicitar desde el propedéutico, el proyecto que buscan trabajar avalado por el académico que lo dirigirá (se debe considerar el perfil del tu- 
tor acorde al tema propuesto), lo cual aunado a las estrategias para dar seguimiento de los avances durante los cuatro semestres previos al egreso, incrementará la posibilidad de completar el proceso formativo y evitará en cierta medida que los posgrados sean utilizados tan sólo como centros de credencialización pedagógica. En relación al tiempo para graduarse, se debe hacer un llamado y explicar la situación académica reglamentaria de cada uno de los egresados; y en su caso se deberá establecer un espacio donde se trabaje con los interesados en graduarse, considerar no volver a masificar la matrícula, ya que en parte esto ha provocado que la MECEA esté por debajo de los porcentajes mínimos de graduación que reportan la Asociación Nacional de Universidades e Instituciones de Educación Superior (ANUIES) y el Consejo Nacional de Ciencia y Tecnología (CONACyT) en México.

\section{Referencias Bibliográficas}

Asociación Nacional de Universidades e Instituciones de Educación Superior (ANUIES) (2011). Anuario estadístico Población escolar y personal docente en la educación media superior y superior ciclo escolar 20102011. México: ANUIES.

Arenas-Castellanos, M.V., \& Fernández de Juan, T. (2009). Formación pedagógica docente y desempeño académico de alumnos en la facultad de ciencias administrativas de la UABC. Revista de la Educación Superior, 38(150), 7-18.

Arredondo, M., \& Santa María M. (2004). El proceso de graduación en programas de posgrado de ciencias experimentales en la UNAM. En M. Arredondo y R. Sánchez Puentes (Coords.), Campo científico y formación en el posgrado (pp. 203-226). México: Universidad Nacional Autónoma de México-Plaza y Valdés.

Auyón-Ley, P., \& Durazo-Durazo, A.L. (2003). El impacto de los egresados del diplomado en educación Ambiental en la práctica educativa del nivel preescolar. (Tesis inédita de maestría). Universidad Pedagógica Nacional. Mexicali, B.C.

Bedoy-Velázquez, V., Castro-Rosales E. A., Romo-Reyes, M. M., Reyes-Ruiz, J., Pérez-Peña, O. \& Ramírez-Quintana, A. I. (2008). La experiencia de una década de la maestría en educación ambiental a distancia de la Univer- sidad de Guadalajara, México. “Hacia un Posgrado de Calidad. In F. Reyes-Escutia \& Ma. T. Bravo-Mercado (Eds.), Educación Ambiental para la sustentabilidad en México Aproximaciones conceptuales, metodológicas y prácticas. (PP. 98-114). Tuxtla Gutiérrez: Universidad de Ciencias y Artes de Chiapas.

Chehaybar, K. E. (2003). Técnicas para el aprendizaje grupal. México: Plaza y Valdés editores.

Consejo Nacional de Ciencia y Tecnología (CONACYT).

Cuéllar-Saavedra, O. \& Bolívar-Espinoza, A.G. (2006). ¿Cómo estimar la eficiencia Terminal en la educación Superior? Notas sobre su Estatuto Teórico. Revista de la Educación Superior. 3(139), 7-27.

Fuentes-Amaya, S. (2002). Educación Ambiental como discurso ético: El programa de Educación Ambiental de Mexicali. En R. N. Buenfil-Burgos (Coord.). Configuraciones Discursivas en el Campo Educativo (123142). México. Plaza y Valdés.

González, G. E. (1998). Centro y Periferia de la Educación Ambiental. México: Mundi Prensa.

Jaik-Dipp, A. \& Quiroga-Sánchez, C. E. (2009). Estudio de las variables relacionadas con la eficiencia terminal desde una perspectiva teórica fundamentada en el comportamiento organizacional. Revista Visión Educativa IUNAES (n.e.) 3(8), 4-15.

Legorreta, Y. (2001). Deserción, rezago y eficiencia terminal en las IES: Factores normativos que obstaculizan el egreso y la titulación. México: ANUIES.

López-Suárez, A., Albíter-Rodríguez, Á. \& Ramírez-Revueltas, L. (2008). Eficiencia terminal en la educación superior: La necesidad de un nuevo Paradigma. Revista de la Educación Superior. 37(146), 135-151.

Molina, H. J.L., Gutiérrez de la Rosa, M.E., Mata, G.E. \& Luna, H.G. (2001). El impacto de la UPN-Unidad Mexicali en la Educación Básica de la Región. Reporte Final de Investigación. Mexicali: SIMAC-CONACYT.

Ramírez-Acosta, J. y Molina-Hernández, J. (2011). Entre la necesidad de credencialización y la vocación de estudios de posgrado. Espíritu Científico en Acción. 14, 3-16.

Sánchez-Dromundo, R. A. (2007). La teoría de los campos de Bourdieu, como esquema teórico de análisis del proceso de graduación en posgrado. Revista Electrónica de Investigación Educativa, 9 (1), 1-21. Recuperado de: http://redie.uabc.mx/vol9no1/contenido/ dromundo.html 
Subsecretaría de Educación Pública (2008). Estudio de la eficiencia terminal de las Instituciones de Educación Superior Mexicanas. Recuperado de http://www.ses. sep.gob.mx/wb/ses/estudio_de_la_eficiencia_terminal_de_las_ies_m

Reglamento interno de la $\mathrm{H}$. Comisión Estatal Mixta de Escalafón, para los trabajadores de la Secretaría de Educación y Bienestar Social (1990). Periódico Oficial, $22 \S$ XCVII.

Universidad Pedagógica Nacional (1993). Plan de Estudios de la Maestría en Educación: Campo Educación
Ambiental. Mexicali.

Universidad Pedagógica Nacional (1999). Reglamento General de Estudios de Posgrado. México. Consejo Académico UPN. México.

Universidad Pedagógica Nacional (2012). Reglamento General de Estudios de Posgrado. México. Consejo Académico UPN. México.

\section{Cómo citar este artículo:}

Ramírez-Acosta, J. (2013). Análisis de la eficacia terminal de la Maestría en Educación: Campo Educación Ambiental de UPN-Mexicali. Espiral, Revista de Docencia e Investigación. 2 (2), 19-36 